\title{
Alodinia é mais frequente nos indivíduos com crises mais intensas de cefaleia e nas mulheres
}

\author{
Allodynia is more frequent in the individuals with more intense attacks \\ of headache and in women
}

\author{
Gêssyca Adryene de Menezes Silva ${ }^{2,3}$, Simone de Siqueira Bringel ${ }^{3}$, Hugo André de Lima Martins ${ }^{2}$, \\ Rosana Christine Cavalcanti Ximenes², Marcelo Moraes Valença², Daniella Araújo de Oliveira ${ }^{1,2}$ \\ 'Departamentos de Fisioterapia e ${ }^{2}$ Neuropsiquiatria, Universidade Federal de Pernambuco (UFPE), Recife, PE, Brasil \\ ${ }^{3}$ Faculdade ASCES - Associação Caruaruense de Ensino Superior, Caruaru, PE, Brasil \\ Silva GA, Bringel SS, Martins HA, Ximenes RC, Valença MM, Oliveira DA \\ [Allodynia is more frequent in the individuals with more intense attacks of headache and in women] \\ Headache Medicine. 2012;3(2):88-91. Portuguese
}

\section{RESUMO}

Objetivo: Identificar a presença de alodinia em alunos com cefaleia primária de uma Instituição de Ensino Superior. Método: Foram avaliados 378 alunos (273 mulheres) com idade entre 18 e 45 anos ( $22 \pm 5$ anos). Foi utilizado um questionário sobre as características clínicas da cefaleia, baseado nos critérios da ICHD-II (2004), e um questionário para identificação e diferenciação da alodinia cefálica e extracefálica. Resultados: Na amostra estudada, $374 / 378$ (98,9\%) dos alunos apresentaram cefaleia ao longo da vida $[271 / 273(99,3 \%)$ mulheres e $103 / 105(98,1 \%)$ homens, $\left.p=0,309 ; \chi^{2}\right]$ e $334 / 378(88,4 \%)$ queixaram-se de cefaleia nos últimos três meses $[248 / 273(90,8 \%)$ mulheres e $86 / 105(81,9 \%)$ homens, $\left.p=0,020 ; \chi^{2}\right]$. Dos alunos com cefaleia nos últimos três meses $331 / 378$ (87,6\%) apresentaram alodinia $[250 / 273(91,6 \%)$ mulheres e $81 / 105(77,1 \%)$ homens, $p<0,001 ; \chi^{2}$. Houve associação entre a intensidade da cefaleia nos últimos três meses e a presença de alodinia [5/ $12(41,7 \%)$ dos indivíduos com dor leve, $211 / 236(89,4 \%)$ dor moderada e 83/86 (96,5\%) dor intensa; $\left.p<0,001 ; \chi^{2}\right]$. A alodinia cefálica foi mais frequente nas seguintes condições: pentear o cabelo $(43,5 \%)$, rabo de cavalo $(57,3 \%)$ nas mulheres; uso de óculos (33,7\%), nos homens; uso de chapéu ou boné $(53,6 \%$ mulheres e $59,3 \%$ homens), exposição ao frio (45,6\% mulheres e $41,9 \%$ homens) e ao calor (56,9\% mulheres e 50\% homens). A alodinia extracefálica foi mais frequentemente desencadeada na exposição ao calor (60,9\% mulheres e 59,3\% homens) e ao frio (42,7 mulheres e 38,4\% homens). Conclusão: Alodinia é mais frequente nas mulheres e em indivíduos durante crises mais intensas de cefaleia.

Palavras-chave: Cefaleia; Migrânea; Alodinia sensitiva

\section{ABSTRACT}

Objective: Identifying the presence of allodynia in students with primary headache in a college. Method: It was evaluated 378 students (273 women) aged between 18 and 45 years (22 \pm 5 years). A questionnaire was used on the clinical characteristics of headache based on ICHD II-2004 criteria, and another one for the identification and differentiation of cephalic and extra-cephalic allodynia. Results: In this sample $374 / 378$ (98.9\%) students had headaches throughout life [271/ $273(99.3 \%)$ females and 103/105 (98.1\%) men, $p=0.309$; $\left.\chi^{2}\right]$ and $334 / 378(88.4 \%)$ complained of headache in the last three months $[248 / 273$ (90.8\%) women and 86/105 (81.9\%) men, $\mathrm{p}=0.020 ; \chi^{2}$. Of the students with headache in the last three months $331 / 378$ (87.6\%) had allodynia [250/273 (91.6\%) women and $81 / 105(77.1 \%)$ men, $p<0.001 ; \chi^{2}$. There was an association between the intensity of the headache in the last three months and the presence of allodynia [5/12 (41.7\%) of the individuals with mild pain, $211 / 236$ (89.4\%) moderate pain and $83 / 86(96.5 \%)$ severe pain; $\left.p<0.001 ; \chi^{2}\right]$. Cephalic allodynia was more frequent in conditions such as combing the hair (43.5) the use of ponytail (57.3\%), use of glasses (33.7\%), use of hat or cap (53.6\% women and $59.3 \%$ man), exposure to coldness (45.6\% women and $41.9 \%$ man) and heat exposure (56.9\% woman and $50 \%$ men). The extracephalic allodynia was more frequently triggered in heat exposure (60.9\% women and $59.3 \%$ men) and coldness (42.7\% women and $38.4 \%$ men). Conclusion: Allodynia is more frequent in women and in individuals with more intense attacks of headache.

Keywords: Headache; Migraine; Sensory allodynia 


\section{INTRODUÇÃO}

A migrânea é um distúrbio comum e complexo do sistema nervoso central que apresenta como sinais e sintomas dor de cabeça, náuseas e sensibilidade a luz, ao som, ao cheiro ou a movimentação da cabeça. ${ }^{(1)} \mathrm{Em}$ indivíduos com cefaleia, em especial migranosos crônicos, a alodinia é frequentemente associada, sendo esta definida como uma percepção de dor ou incômodo ao receber estímulos não dolorosos táteis ou térmicos na pele normal, durante atividades cotidianas. ${ }^{(2,3)}$

A alodinia, em migranosos, resulta de uma alteração na regulação da via nociceptiva central.(2) $\bigcirc$ gênero feminino e a frequência das crises de cefaleia também possuem uma forte associação com presença de alodinia nesses pacientes. ${ }^{(2,4)}$

A alodinia pode se apresentar durante ou após os episódios de migrânea e os indivíduos que a apresentam tornam-se incapazes de executar qualquer ação que envolva o toque na região do rosto, do couro cabeludo ou em algumas regiões corporais. Estudos demonstram que se pode interpretar a alodinia como um marcador da frequência da migrânea e está presente em $80 \%$ dos casos, nas fases posteriores de um ataque agudo. $(5,6)$

A alodinia, durante uma crise de migrânea, geralmente se distribui na região álgica, mas também pode estar presente em outras áreas cefálicas ou extracefálicas. ${ }^{(2)}$ A alodinia cefálica acomete a região da cabeça e tem como principais sintomas referidos pelos indivíduos: dor ao pentear o cabelo, barbear o rosto, uso de óculos, uso de brincos, colares, chapéus, toucas ou atacas de cabelos. Já a extracefálica é caracterizada com dor ou sensação desagradável em uma região corpórea durante uma crise de cefaleia, isso ocorre ao usar roupas apertadas, relógio, pulseira, ao tomar banho, exposição ao calor ou frio. ${ }^{(7)}$

Desta maneira, tanto a alodinia quanto a cefaleia (migrânea) podem influenciar negativamente o bem-estar e cotidiano do indivíduo, o que acarreta um grande impacto socioeconômico, uma vez que há uma redução da sua produtividade, aumento nos custos referentes aos serviços de saúde, absenteísmo trabalhista, comprometimento do estado psicoafetivo, dentre outras, determinando assim prejuízos diretos na sua qualidade de vida. ${ }^{(8-10)}$

O objetivo desse estudo é avaliar a presença de alodinia sensitiva em estudantes com cefaleia de uma instituição de ensino superior.

\section{MÉTODO}

Este estudo foi realizado na Faculdade ASCES - Associação Caruaruense de Ensino Superior, Caruaru, PE, Brasil. Trata-se de um estudo corte transversal realizado no período de agosto de 2010 a fevereiro de 2011 . Foram entrevistados 378 alunos (273 mulheres), regularmente matriculados, com idade entre 18 e 45 anos ( $22 \pm 5$ anos). Foram excluídos indivíduos com doença neurológica conhecida e aqueles com algum tipo de cefaleia secundária. Todos os participantes responderam a um formulário contendo informações sociodemográficas.

Para diagnóstico do tipo de cefaleia foi utilizado os critérios da International Headache Society - IHS (ICHD-II, 2004).. (1) Alunos que apresentavam tanto cefaleia do tipo tensional (CTT) quanto migrânea foram incluídos no grupo de migranosos. Para avaliar a alodinia foi utilizado um questionário adaptado que identifica e diferencia a alodinia em cefálica e extracefálica. ${ }^{(12)} \mathrm{A}$ intensidade da dor foi quantificada pela escala numérica de dor (0-10).

O questionário de alodinia é dividido em duas partes: para avaliar a alodinia cefálica o questionário é composto por 13 questões relativas ao indivíduo apresentar dor ou alguma sensação desagradável quando realiza atividades cotidianas como pentear o cabelo, usar rabo de cavalo, se expor ao calor e/ou ao frio, dentre outras; e para a extracefálica, sete questões, que identificam se o indivíduo possui sensação desagradável ou dor ao usar roupas apertadas ou objetos no punho, tomar banho, dentre outras. As respostas foram estruturadas de forma dicotômica, lidas e respondidas pelo próprio voluntário.

Os dados obtidos na pesquisa são mostrados como média \pm erro padrão. Utilizamos o teste KolmogorovSmirnov para verificar o tipo de distribuição das variáveis a serem estudadas. Para as variáveis que não apresentaram uma distribuição normal utilizamos o teste não paramétrico de Mann-Whitney. Na análise das variáveis categóricas aplicamos o qui-quadrado $\left(\chi^{2}\right)$ ou o teste exato de Fisher, conforme a frequência esperada nas células. O nível de significância considerado como diferente estatisticamente foi $p<0,05$. Para o processamento e análise dos dados foi o utilizado o programa GraphPad Prism versão 5.0.

\section{RESULTADOS}

Na amostra estudada 374/378 (98,9\%) dos alunos referiram cefaleia ao longo da vida $[271 / 273(99,3 \%)$ mulheres e 103/105 (98,1\%) homens, $\left.\mathrm{p}=0,309 ; \chi^{2}\right]$ e 
$334 / 378(88,4 \%)$ queixaram-se de cefaleia nos últimos três meses [248/273 (90,8\%) mulheres e 86/105 (81,9\%) homens, $p=0,020 ; \chi^{2}$. Dos alunos com cefaleia nos últimos três meses $331 / 378(87,6 \%)$ apresentaram alodinia $[250 / 273(91,6 \%)$ mulheres e $81 / 105(77,1 \%)$ homens], $p<0,001 ; \chi^{2}$. Houve associação entre a intensidade da cefaleia nos últimos três meses e a presença de alodinia $[5 / 12(41,7 \%)$ dos indivíduos com dor leve e 83/86 (96,5\%) dor intensa; OR 38,7 (IC 95\% 7,6 197), $\mathrm{p}<0,001$ ] (Figura 1).

As Tabelas 1 e 2 mostram a distribuição percentual das condições que desencadeiam cada uma das formas de alodinia, cefálica e extracefálica, respectivamente.

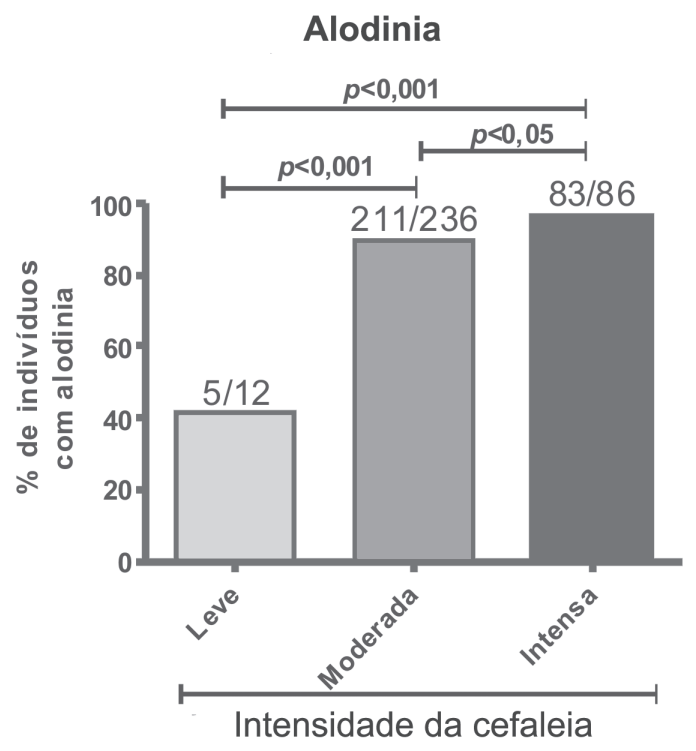

Figura 1. Presença de alodinia nos indivíduos com cefaleia de acordo com a intensidade das crises de cefaleia. Comparações estatísticas utilizando o teste exato de Fisher.

Tabela 1. Distribuição percentual das condições que desencadearam alodinia cefálica entre os gêneros nos 331 alunos com cefaleia

\begin{tabular}{lrr}
\hline $\begin{array}{l}\text { Condições que desencadearam alodinia } \\
\text { cefálica }\end{array}$ & Mulheres \% & Homens \% \\
\hline Pentear o cabelo & 43,5 & 11,6 \\
Uso de rabo de cavalo & 53,7 & - \\
Barbear-se & - & 14,0 \\
Usar óculos & 35,5 & 3,7 \\
Usar lentes de contato & 14,5 & 12,8 \\
Usar brincos & 16,1 & 10,5 \\
Usar cordão no pescoço & 24,6 & 15,1 \\
Usar chapéu ou boné & 53,6 & 59,3 \\
Usar anel no dedo & 14,1 & 9,3 \\
Lavar o rosto & 17,3 & 14,0 \\
Colocar a cabeça no travesseiro & 28,6 & 29,1 \\
Expor-se ao calor & 56,9 & 50,0 \\
Expor-se ao frio & 45,6 & 41,9 \\
\hline
\end{tabular}

A alodinia cefálica foi mais frequente nas condições: pentear o cabelo $(43,5 \%)$, rabo de cavalo $(57,3 \%)$, nas mulheres; uso de óculos $(33,7 \%)$, nos homens; uso de chapéu ou boné $(53,6 \%$ mulheres e $59,3 \%$ homens), exposição ao frio ( $45,6 \%$ mulheres e $41,9 \%$ homens) e ao calor (56,9\% mulheres e 50\% homens). A alodinia extracefálica foi mais frequentemente desencadeada na exposição ao calor (60,9\% mulheres e $59,3 \%$ homens) e ao frio $(42,7$ mulheres e $38,4 \%$ homens).

Tabela 2 - Distribuição percentual das condições que desencadearam alodinia extracefálica entre os gêneros nos 331 alunos com cefaleia

\begin{tabular}{lcc}
\hline $\begin{array}{l}\text { Condições que desencadearam alodinia } \\
\text { extracefálica }\end{array}$ & Mulheres \% & Homens \% \\
\hline Usar roupas apertadas & 35,0 & 30,2 \\
Usar algum objeto no punho (relógio, & 15,3 & 16,3 \\
pulseiras) & 12,5 & 15,1 \\
Cobrir-se com um cobertor mais pesado & 14,5 & 15,1 \\
Tomar banho & 60,9 & 59,3 \\
Expor-se ao calor & 42,7 & 38,4 \\
Expor-se ao frio &
\end{tabular}

\section{DISCUSSÃO}

No presente estudo, nos 378 voluntários a porcentagem de mulheres afetadas com cefaleia primária e alodinia foi mais elevada quando comparado ao sexo oposto (91,6\% e 77,1\%, respectivamente), havendo consenso com outros autores, ${ }^{(2,13-15)}$ que também acharam em seus estudos altos índices para tais variáveis. Estudos demonstram que as mulheres apresentam até quatro vezes mais risco de ter cefaleia do que os homens, sendo esse achado unânime na literatura e é possível que a sua explicação seja decorrente das variações hormonais que as mulheres sofrem no decorrer do ciclo menstrual. ${ }^{(13,16)}$

Estudos mostram que a ação de hormônios no sistema reprodutor feminino, na dor, pode em parte explicar a maior prevalência de sintomas de alodinia sensorial e outras síndromes dolorosas nas mulheres, havendo assim uma associação da mesma com o efeito hormonal. ${ }^{(12)}$ Tal opinião é corroborada por outros estudos que mostram que os níveis de dor na cefaleia e outros distúrbios álgicos, aparecem por todo ciclo menstrual. ${ }^{(12,17-19)}$

O estudo de Stewart et al. (1995), ${ }^{(20)}$ realizado através de meta-análise, mostrou que o sexo e a idade são fatores responsáveis pela variação nos achados de prevalência da cefaleia. Nesse estudo, os autores apontaram que o gênero é responsável por 15\% dessa variabilidade e somado à idade corresponde por $30 \%$ de tais diferenças. ${ }^{(8,20)}$ 
A alodinia associada a cefaleia vem atraindo interesse por parte dos pesquisadores, visto que é reconhecida como um sinal da sensibilização central durante episódios de cefaleia e fator de risco para a progressão da cefaleia crônica. ${ }^{(7)}$ Com relação à intensidade dolorosa, neste estudo, observou-se que indivíduos que têm cefaleia e alodinia possuem uma maior tendência a dor de moderada a intensa. Estudos com testes sensorias e/ou dados extraídos de questionários sugerem que até $80 \%$ dos pacientes com cefaleia possuem crises de alodinia associadas. ${ }^{(17,19,21,22)}$ Tais estudos coincidem com o resultado obtido neste trabalho.

O presente estudo encontrou um grande percentual de indivíduos com cefaleia e da mesma associada com alodinia em indivíduos do sexo feminino estando de acordo com a literatura. Indivíduos com cefaleia além do sofrimento individual apresentam um prejuízo econômico de custos diretos (atenção médica e medicamentosa) e indiretos (redução da sua produtividade, absenteísmo e até mesmo incapacidade durante as crises) o que favorece o comprometimento global da sua qualidade de vida. Diante do exposto, é imprescindível à conscientização e educação a respeito do tema, alertando para a grande importância de uma profilaxia, tratamento e acompanhamento médico, multi e interprofissional adequado, corroborando assim na efetiva redução da frequência e intensidade da cefaleia o que reflete positivamente no bem-estar, na relação custo-benefício, na produtividade e melhora da qualidade de vida do indivíduo.

\section{REFERÊNCIAS}

1. Monteiro JMP. Cefaleias Primárias: causas e consequências. Rev Port Clin Geral 2006;22:455-9.

2. Lovati C, D'Amico D, Bertora P. Allodynia in migraine: frequent random association or unavoidable consequence? Expert Rev Neurother. 2009;9(3):395-408.

3. Burstein R, Yarnitsky D, Goor-Aryeh I, Ransil BJ, Bajwa ZH . An association between migraine and cutaneous allodynia. Ann Neurol. 2000;47(5):614-24.

4. Bigal ME, Ashina S, Burstein R, Reed ML, Buse D, Serrano D, Lipton RB; AMPP Group. Prevalence and characteristics of allodynia in headache sufferers: a population study. Neurology. 2008;70 (17):1525-33.

5. Cuadrado ML, Young WB, Fernández-de-las-Peñas C, Arias JA, Pareja JA. Migrainous corpalgia: body pain and allodynia associated with migraine attacks. Cephalalgia. 2008;28(1):87-91 .

6. Silberstein SD. Migraine pathophysiology and its clinical implications. Cephalalgia. 2004;24(Suppl 2):2-7.

7. Guy N, Marques AR, Orliaguet T, Lanteri-Minet M, Dallel R, Clavelou P. Are there differences between cephalic and extracephalic cutaneous allodynia in migraine patients? Cephalalgia. 2010;30(7):881-6.
8. Vincent M, Rodrigues Ade J, De Oliveira GV, De Souza KF, Doi $L M$, Rocha MB, et al. Prevalence and indirect costs of headache in a Brazilian Company. Arq Neuropsiquiatr. 1998;56(4):734-43.

9. Oliveira, DA, Silva LC, Brito JKC, Aleixo JD, Silva EIM, Valença MM. O impacto da migrânea nas atividades de vida diária é mais incapacitante nas mulheres. Migrâneas \& Cefaleias. 2008; $11(4): 252-4$.

10. Oliveira DA, Brito JKC, Souza CMS, Cruz CKR, Silva LC, Siqueira GR, et al. Cefaleia do tipo tensional e migrânea em funcionários de uma instituição de ensino superior: grau de incapacidade. Headache Medicine. 2011 ;2(2):61-5.

1 1. ICHD - II Headache Classification Subcommittee of the International Headache Society. The International Classification of Headache Disorders: 2nd edition. Cephalalgia. 2004;24 (Supl 1):9-160.

12. Lipton RB, Bigal ME, Ashina S, Burstein R, Silberstein S, Reed ML, Serrano D, Stewart WF; American Migraine Prevalence Prevention Advisory Group. Cutaneous allodynia in the migraine population. Ann Neurol. 2008;63(2):148-58. Comment on: Ann Neurol. 2008; 63(2):130-2.

13. Pahim LS, Menezes AMB, Lima R. Prevalence and factors associated to migraine in adult population, Southern Brazil. Rev Saude Pública. 2006;40:1-7. Portuguese.

14. Henry P, Auray JP, Gaudin AF, Dartigues JF, Duru G, Lantéri-Minet $M$, et al. Prevalence and clinical characteristics of migraine in France. Neurology. 2002;59(2):232-7.

15. Rasmussen BK. Epidemiology of migraine. Biomed Pharmacother. 1995;49(10):452-5.

16. Stewart WF, Lipton RB, Liberman J. Variation in migraine prevalence by race. Neurology. 1996;47(1):52-9.

17. Piovesan EJ, Giublin ML, Werneck LC. Distúrbios sensoriais associados à migrânea. Relato de caso e revisão da literatura. Rev Dor. 2005;6:666-71.

18. Heitkemper MM, Jarrett M. Pattern of gastrointestinal and somatic symptoms across the menstrual cycle. Gastroenterology. 1992; 102(2):505- 13.

19. MacGregor EA. Menstrual migraine. Curr Opin Neurol. 2008; $21(3): 309-15$.

20. Stewart WF, Simon D, Shechter A, Lipton RB. Population variation in migraine prevalence: a meta-analysis. J Clin Epidemiol. 1995; 48(2):269-80.

21. Burstein R, Yarnitsky D, Goor-Aryeh I, Ransil BJ, Bajwa ZH. An association between migraine and cutaneous allodynia. Ann Neurol. 2000;47(5):614-24.

22. Tietjen GE, Brandes JL, Peterlin BL, Eloff A, Dafer RM, Stein MR, et al. Allodynia in migraine: association with comorbid pain conditions. Headache. 2009;49(9):1333-44.

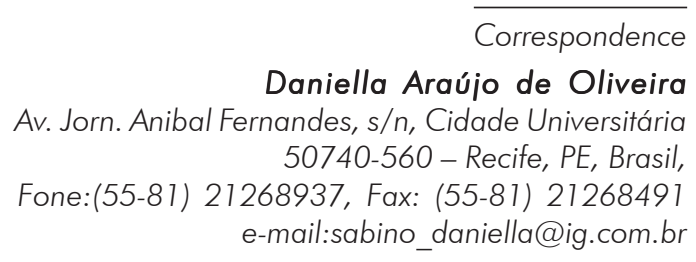

Recebido: 5/2/2012

Aceito: 14/6/2012 